\title{
Protein Configuration Landscape Fluctuations Revealed by Exciton Transition Polarizations in Single Light Harvesting Complexes. Supporting Information
}

\author{
Sumera Tubasum ${ }^{1}$, Magne Torbjörnsson*1,2, Dheerendra Yadav $^{1}$, Rafael \\ Camacho $^{1}$, Gustaf Söderlind ${ }^{2}$, Ivan G Scheblykin ${ }^{1}$, and Tõnu Pullerits ${ }^{1}$ \\ ${ }^{1}$ Division of Chemical Physics, Department of Chemistry, \\ Lund University \\ ${ }^{2}$ Division of Numerical Analysis, Centre for Mathematical Sciences, \\ Lund University
}

Here we provide mathematical and programming details about the modelling work in the paper. For details about the experimental setup and early modelling please see ref. [1]. The model described below simulates $2 D$ polarization imaging (2D-POLIM) spectra using Redfield theory to model energy relaxation within a single light harvesting complex consisting of $N$ pigment molecules represented as $N$ two-level system with transition dipole moments $\bar{\mu}$. The final 2D-POLIM spectra, $\bar{F}$, will be $m \times m$ matrixes.

\section{Simulation of 2D Polarization Single Molecule Imaging Spectra}

\subsection{Model Explanation}

The aim of this text is to explain in detail the model used to simulate a 2D-POLIM Spectrum.

The experimental results are expressed as 2D plots with the excitation polarization angle on the $x$-axis, $\varphi_{e x}$, and the emission polarization angle, $\varphi_{e m}$, on the $y$-axis. The colour of each point shows the intensity of the emission with polarization $\varphi_{e m}$ after absorbed at $\varphi_{e x}$. This can be understood by using the well known fact that a multimolecular complex can be treated as a system of many transition dipoles, where the

*Modelling work, contact: magne.torbjornsson@chemphys.lu.se 
probability that each of these transition dipoles absorbs or emits polarized light are given by, [2]:

$$
P_{\text {Abs or em }} \propto|\hat{\mu} \cdot \bar{e}|^{2},
$$

where $\bar{e}$ is the polarization vector of the laser or the measurement and is given by $\bar{e}(\varphi)=$ $[\cos (\varphi), \sin (\varphi), 0]$. The probability (or intensity) that the transition dipole $\alpha$ absorbs energy, and that the energy transfers to and is emitted from transition dipole $\beta$, can therefore be written as follows:

$$
P_{\alpha \rightarrow \beta} \propto\left|\hat{\mu}_{\alpha} \cdot \bar{e}\left(\varphi_{e x}\right)\right|^{2} \tilde{G}_{\beta, \alpha}\left|\hat{\mu}_{\beta} \cdot \bar{e}\left(\varphi_{e m}\right)\right|^{2},
$$

where $\tilde{G}_{\beta, \alpha}$ is the probability for energy transfer from $\alpha$ to $\beta$, [3]. The total 2D spectrum is given by summing over all transition dipoles in the molecule, [4]:

$$
F\left(\varphi_{e x}, \varphi_{e m}, E_{e x}, E_{e m}\right)=\sum_{\alpha, \beta}\left|\hat{\mu}_{\alpha} \cdot \bar{e}\left(\varphi_{e x}\right)\right|^{2} f_{\alpha}^{e x}\left(E_{e x}\right) \tilde{G}_{\beta, \alpha}\left|\hat{\mu}_{\beta} \cdot \bar{e}\left(\varphi_{e m}\right)\right|^{2} f_{\beta}^{e m}\left(E_{e m}\right),
$$

where $E_{e x}$ and $E_{e m}$ are, respectively, the energy of the excitation laser, and the energy for which the emission is measured. In the experimental setup used to obtain the data, the emission part is integrated over all energies as well as over time. The lowest detected energy is here defined to be zero, and the highest energy is denoted $E_{f}$. The highest energy is experimentally given by a sharp cut-off filter, so choosing $E_{f}$ as the upper integral limit is sufficient to model the filter.

$$
F\left(\varphi_{e x}, \varphi_{e m}, E_{e x}\right)=\sum_{\alpha, \beta}\left(\left|\hat{\mu}_{\alpha} \cdot \bar{e}\left(\varphi_{e x}\right)\right|^{2} f_{\alpha}^{e x}\left(E_{e x}\right) \tilde{G}_{\beta, \alpha}\left|\hat{\mu}_{\beta} \cdot \bar{e}\left(\varphi_{e m}\right)\right|^{2} \int_{0}^{E_{f}} f_{\beta}^{e m}\left(E_{e m}\right) \mathrm{d} E_{e m}\right)
$$

The functions $f(E)$ are line broadening functions included to model experimental uncertainties, and chosen as, [5]:

$$
\begin{aligned}
f_{\alpha}^{e m}(E) & =\frac{1}{\sqrt{2 \pi} \sigma} \exp \left(\frac{-\left(E-\mathcal{E}_{\alpha}+\delta_{\text {em }}\right)^{2}}{2 \sigma_{\alpha}^{2}}\right), \\
f_{\alpha}^{e x}(E) & =\frac{1}{\sqrt{2 \pi} \sigma} \exp \left(\frac{-\left(E-\mathcal{E}_{\alpha}+\delta_{e x}\right)^{2}}{2 \sigma_{\alpha}^{2}}\right) .
\end{aligned}
$$

Here $\mathcal{E}_{\alpha}$ are the eigenvalues of the Hamiltonian (the allowed discrete energy levels) given below, and control the centering of the the width of the standard deviation curve $\sigma$. The parameters $\delta$ model a Stokes shift.

\subsection{Quantum Mechanics and System Description}

A molecule complex can be described quantum mechanically by using a Frenckel exciton Hamiltonian, [6]. In this calculation only electronic transition for each molecule is used, giving the Hamiltonian

$$
H_{e x}=\sum_{i}\left(E+D_{i}+\Delta_{i}\right)|i\rangle\left\langle i\left|+\sum_{i, j} V_{i, j}\right| i\right\rangle\langle j|,
$$


where $E$ is the energy of a free molecule, $D_{i}$ is a systematic shift caused by the molecule's environment, and $\Delta_{i}$ is a random inhomogeneous distribution shift assumed to have a normal distribution. In the following calculations $H_{e x}$ is given in matrix form, $\bar{H}_{e x}$. Reading (7) as a matrix can be done by realizing that the outer product $|i\rangle\langle j|$ corresponds to the $i, j$ 'th element of $\bar{H}_{e x} . \bar{H}_{e x}$ will therefore be an $N \times N$ matrix with molecular energies on the diagonal and the coupling between the molecules as off-diagonal elements. The coupling $V_{i, j}$ is of dipole-dipole type and can be calculated as

$$
V_{i, j}=\frac{1}{4 \pi \epsilon_{0}}\left(\frac{\bar{\mu}_{i} \cdot \bar{\mu}_{j}}{r_{i, j}^{3}}-3 \frac{\left(\bar{r}_{i, j} \cdot \bar{\mu}_{i}\right)\left(\bar{r}_{i, j} \cdot \bar{\mu}_{j}\right)}{r_{i, j}^{5}}\right),
$$

where $\bar{r}_{i, j}$ is the vector between the dipoles $\bar{\mu}_{i}$ and $\bar{\mu}_{j}$ and $r_{i, j}$ is their distance. The excitonic states are given by solving the eigenvalue problem

$$
\bar{H}_{e x}|\alpha\rangle=\mathcal{E}_{\alpha}|\alpha\rangle
$$

where $|\alpha\rangle=\left[C_{1, \alpha}, C_{2, \alpha}, \ldots, C_{N, \alpha}\right]^{\mathrm{T}}$ denotes an $N \times 1$ vector and $\mathcal{E}_{\alpha}$ an eigenenergy. To model the entire complex it is necessary to calculate the transition dipoles. The transition dipole corresponding to the state $|\alpha\rangle$ is given by

$$
\hat{\mu}_{\alpha}=\sum_{i} \bar{\mu}_{i} C_{i, \alpha}
$$

Defining $\bar{C}=[|1\rangle, \ldots,|N\rangle],(N \times N)$, and $\overline{\mathcal{E}}=\left[\mathcal{E}_{1}, \mathcal{E}_{2}, \ldots, \mathcal{E}_{N}\right]^{\mathrm{T}},(N \times 1),(10)$ can be written as

$$
\hat{\mu}=\bar{\mu} \bar{C}
$$

where

$$
\bar{\mu}=\left[\begin{array}{lll}
\mu_{x, 1} & \cdots & \mu_{x, N} \\
\mu_{y, 1} & \cdots & \mu_{y, N} \\
\mu_{z, 1} & \cdots & \mu_{z, N}
\end{array}\right] .
$$

To simulate that the complex can have different orientations, the transition dipoles are rotated with a rotation matrix $R_{\text {rot }}^{\theta}$, giving

$$
\hat{\mu}^{\theta}=\bar{R}_{r o t}^{\theta} \hat{\mu}
$$

where $R_{\text {rot }}^{\theta}$ is chosen to only rotate the molecule complex around a single axis by an angle of $\theta$.

\subsection{Energy Transfer Rates}

Starting from the expression for the transfer rates given by Redfield Theory, the energy transfer rates from state $\alpha$ to $\beta, W_{\beta, \alpha}$, used in (22) to calculate the exciton population of the states, are given by

$$
W_{\beta, \alpha}=W^{+}\left(\mathcal{E}_{\beta, \alpha}\right) \sum_{k}\left|C_{k, \alpha}\right|^{2}\left|C_{k, \beta}\right|^{2}
$$


for positive values of the energy difference $\mathcal{E}_{\beta, \alpha}$ between the states $\alpha$ and $\beta$, and by

$$
W_{\beta, \alpha}=W^{-}\left(\mathcal{E}_{\beta, \alpha}\right) \sum_{k}\left|C_{k, \alpha}\right|^{2}\left|C_{k, \beta}\right|^{2}
$$

for negative energy differences, [7]. The quantities $W^{+}(\mathcal{E})$ and $W^{-}(\mathcal{E})$ are given by

$$
W^{+}(\mathcal{E})=\frac{2 \pi}{\hbar}\left(\frac{1}{\exp (\mathcal{E} / k T)-1}+1\right) \cdot \frac{\mathcal{E}^{2} \exp \left(-\mathcal{E} / E_{0}\right)}{2 E_{0}^{3}} \cdot \mathcal{E}^{2}
$$

for positive values of the energy difference between the states, $\mathcal{E}_{\beta, \alpha}>0$, which corresponds to a downhill transfer, [7]. For negative $\mathcal{E}_{\beta, \alpha}$ (uphill relaxation) the relation is, [7]:

$$
W^{-}(\mathcal{E})=\frac{2 \pi}{\hbar}\left(\frac{1}{\exp (-\mathcal{E} / k T)-1}\right) \cdot \frac{\mathcal{E}^{2} \exp \left(\mathcal{E} / E_{\mathrm{o}}\right)}{2 E_{0}^{3}} \cdot \mathcal{E}^{2} .
$$

Since the energy states are given by the eigenvalues of the Hamiltonian, the energy difference corresponding to transfer from $\alpha$ to $\beta$ are defined as $\mathcal{E}_{\beta, \alpha}=\mathcal{E}_{\alpha}-\mathcal{E}_{\beta}$, [8]. $\mathcal{E}_{\alpha}$ and $\mathcal{E}_{\beta}$ are the eigenvalues of $\bar{H}_{e x}$. To be able to efficiently calculate (14) and (15), the matrix $\Delta \overline{\mathcal{E}}(N \times N)$ is defined as

$$
\Delta \overline{\mathcal{E}}=\left[\begin{array}{ccc}
\mathcal{E}_{1}-\mathcal{E}_{1} & \ldots & \mathcal{E}_{1}-\mathcal{E}_{N} \\
\vdots & \ddots & \vdots \\
\mathcal{E}_{N}-\mathcal{E}_{1} & \ldots & \mathcal{E}_{N}-\mathcal{E}_{N}
\end{array}\right]
$$

and calculated as

$$
\overline{\mathcal{E}} \overline{\mathcal{I}}-\overline{\mathcal{I}}^{\mathrm{T}} \overline{\mathcal{E}}^{\mathrm{T}}
$$

where $\overline{\mathcal{I}}$ is a $1 \times N$ vector with ones. Since $\mathcal{E}_{1}<\mathcal{E}_{2}, \ldots,<\mathcal{E}_{N}, \Delta \overline{\mathcal{E}}$ will have positive values below the diagonal, zeros on the diagonal and negative values above the diagonal. Therefore $\Delta \overline{\mathcal{E}}$ can be split into $\Delta \overline{\mathcal{E}}=\Delta \overline{\mathcal{E}}^{+}+\Delta \overline{\mathcal{E}}^{-}$where $\Delta \overline{\mathcal{E}}^{-}$is an upper triangular matrix with negative values and $\Delta \mathcal{E}^{+}$is a lower triangular matrix with positive values. Then the matrix with the transfer rates is given by:

$$
\bar{W}=\left(W^{+}\left(\Delta \overline{\mathcal{E}}^{+}\right)+W^{-}\left(\Delta \overline{\mathcal{E}}^{-}\right)\right) \cdot * \bar{C}^{\Sigma, s},
$$

where

$$
\bar{C}^{\Sigma, s}=\bar{C}^{s \mathrm{~T}} \bar{C}^{s} .
$$

$\bar{C}^{s}$ is an $N \times N$ matrix with elements $C_{\beta, \alpha}^{s}=\left|C_{\beta, \alpha}\right|^{2}$. Each element in (21), $C_{\beta, \alpha}^{\Sigma, s}$, is the sum in the expressions (14) and (15). The notation $* *$ denotes an element-wise multiplication of the two matrices. Each element $W_{\beta, \alpha}$ in $\bar{W}$ is a transfer rate. The diagonal is zero since there is no transfer rate within a state.

\subsection{Energy Transfer}

The numerical approach to solve the kinetic equations has been earlier used in e.g. [3] and [9]. The Pauli master equation describing energy transfer between the states $|\alpha\rangle$ and $|\beta\rangle$,

$$
\dot{p}_{\alpha}=\sum_{\beta}\left(W_{\alpha, \beta} p_{\beta}-W_{\beta, \alpha} p_{\alpha}\right)-p_{\alpha} / \tau_{e x c}
$$


can be written in matrix form as

$$
\dot{P}=\bar{R} \bar{P}
$$

where $\dot{P}=\left[\dot{p}_{\alpha}, \dot{p}_{\beta}, \ldots, \dot{p}_{N}\right]^{\mathrm{T}}, \bar{P}=\left[p_{\alpha}, p_{\beta}, \ldots, p_{N}\right]^{\mathrm{T}}$ and $\bar{R}$ is a $N \times N$ matrix with the coefficients from (22),

$$
\bar{R}=\bar{W} \cdot *(\overline{\mathcal{I}}-\bar{I})-((\overline{\mathcal{I}}-\bar{I}) \bar{W}) \cdot * \bar{I}-\left(1 / \tau_{\text {exc }}\right) \bar{I},
$$

where $\overline{\mathcal{I}}$ denotes an $N \times N$ matrix of ones, and $\bar{I}$ is an $N \times N$ identity matrix. Using a Green's function approach, the solution to (23) is given by

$$
\bar{P}(t)=\bar{G}(t) \bar{P}(0) \text {. }
$$

Integrating $\bar{G}(t)$ with respect to time gives the matrix $\tilde{G}$ used later in the model to simulate a $2 \mathrm{D}$ spectrum. $\bar{G}(t)$ can be diagonalized as

$$
\bar{G}(t)=\bar{T} \exp (\bar{D} t) \bar{T}^{-1},
$$

where $\bar{T}$ is a matrix containing the eigenvectors of $\bar{R}$ as columns, and $\bar{D}$ is a diagonal matrix with the corresponding eigenvalues on the diagonals. The matrix containing the transfer probabilities, $\tilde{G}$, is given by integration of (26) as

$$
\tilde{G}=\int_{0}^{\infty} \bar{G}(t) \mathrm{d} t=\bar{T} \int_{0}^{\infty} \exp (\bar{D} t) \mathrm{d} t \bar{T}^{-1}=-\bar{T} \bar{D}^{-1} \bar{T}^{-1},
$$

assuming that all eigenvalues of $\bar{R}$ are negative.

\subsection{Fluorescence Spectra}

The 2D-POLIM spectrum will be an $m \times m$ matrix $\bar{F}$ where the elements correspond to the fluorescence for a certain value of the polarization angles $\varphi_{e x}$ and $\varphi_{e m}$ and for a given excitation energy $E_{e x}$,

$$
\bar{F}=\left[\begin{array}{ccc}
F\left(\varphi_{e x, 1}, \varphi_{e m, 1}, E_{e x}\right) & \ldots & F\left(\varphi_{e x, m}, \varphi_{e m, 1}, E_{e x}\right) \\
\vdots & \ddots & \vdots \\
F\left(\varphi_{e x, 1}, \varphi_{e m, m}, E_{e x}\right) & \ldots & F\left(\varphi_{e x, m}, \varphi_{e m, m}, E_{e x}\right)
\end{array}\right]
$$

$\bar{F}$ can be calculated as

$$
\bar{F}=\bar{I}_{i n t} \tilde{G} \bar{A}^{\mathrm{T}}
$$

where $\tilde{G}$ is the $(N \times N)$ matrix with the probabilities for the exciton movement. The matrices $\bar{I}_{\text {int }}(m \times N)$ and $\bar{A}(m \times N)$ are calculated as

$$
\begin{gathered}
\bar{A}=\left[\begin{array}{ccc}
\left|\hat{\mu}_{1}^{\theta} \cdot \bar{e}_{1}\right|^{2} f_{1}^{e x}\left(E_{e x}\right) & \ldots & \left|\hat{\mu}_{N}^{\theta} \cdot \bar{e}_{1}\right|^{2} f_{N}^{e x}\left(E_{e x}\right) \\
\vdots & \ddots & \vdots \\
\left|\hat{\mu}_{1}^{\theta} \cdot \bar{e}_{m}\right|^{2} f_{1}^{e x}\left(E_{e x}\right) & \ldots & \left|\hat{\mu}_{N}^{\theta} \cdot \bar{e}_{m}\right|^{2} f_{N}^{e x}\left(E_{e x}\right)
\end{array}\right], \\
\bar{I}_{\text {int }}=\left[\begin{array}{ccc}
\left|\hat{\mu}_{1}^{\theta} \cdot \bar{e}_{1}\right|^{2} \int f_{1}^{e m} \mathrm{~d} E & \ldots & \left|\hat{\mu}_{N}^{\theta} \cdot \bar{e}_{1}\right|^{2} \int f_{N}^{e m} \mathrm{~d} E \\
\vdots & \ddots & \vdots \\
\left|\hat{\mu}_{1}^{\theta} \cdot \bar{e}_{m}\right|^{2} \int f_{1}^{e m} \mathrm{~d} E & \ldots & \left|\hat{\mu}_{N}^{\theta} \cdot \bar{e}_{m}\right|^{2} \int f_{N}^{e m} \mathrm{~d} E
\end{array}\right] .
\end{gathered}
$$


The matrices $\bar{A}$ and $\bar{I}_{\text {int }}$ can be split in two parts. The first, $\bar{A}_{\mu e}(m \times N)$, only depends on the polarization angles and the second, $\bar{f},(N \times N)$ only depends on the energy states $\mathcal{E}_{\alpha}$, and an energy $E$, which is the energy value for which the broadening functions are calculated. $\bar{A}$ and $\bar{I}_{\text {int }}$ can be written as

$$
\begin{gathered}
\bar{A}=\bar{A}_{\mu e} \bar{f}_{e x}, \\
\bar{I}_{i n t}=\bar{A}_{\mu e} \bar{f}_{i n t},
\end{gathered}
$$

where

$$
\bar{A}_{\mu e}=\left[\begin{array}{ccc}
\left|\hat{\mu}_{1}^{\theta} \cdot \bar{e}_{1}\right|^{2} & \ldots & \left|\hat{\mu}_{N}^{\theta} \cdot \bar{e}_{1}\right|^{2} \\
\vdots & \ddots & \vdots \\
\left|\hat{\mu}_{1}^{\theta} \cdot \bar{e}_{m}\right|^{2} & \ldots & \left|\hat{\mu}_{N}^{\theta} \cdot \bar{e}_{m}\right|^{2}
\end{array}\right]
$$

and

$$
\begin{gathered}
\bar{f}_{e x}=\left[\begin{array}{ccc}
f_{1}^{e x}\left(E_{e x}\right) & \ldots & 0 \\
\vdots & \ddots & \vdots \\
0 & \ldots & f_{N}^{e x}\left(E_{e x}\right)
\end{array}\right] \\
\bar{f}_{\text {int }}=\left[\begin{array}{ccc}
\int f_{1}^{e m}(E) \mathrm{d} E & \ldots & 0 \\
\vdots & \ddots & \vdots \\
0 & \ldots & \int f_{N}^{e m}(E) \mathrm{d} E
\end{array}\right] .
\end{gathered}
$$

To model the dependence of the polarization angles, we introduce the polarization vector $\bar{e}=[\cos (\varphi), \sin (\varphi), 0]$ and a vector with all polarization angles,

$$
\bar{\varphi}=\left[\varphi_{1}, \ldots, \varphi_{m}\right]^{\mathrm{T}} .
$$

It is only necessary to do this once since the fluorescence spectra will have the same angles in absorption as well as emission. Then a matrix with the polarization vectors $\bar{e}=\left[\bar{e}_{1}, \ldots, \bar{e}_{m}\right]^{\mathrm{T}}(m \times 3)$ can be calculated as

$$
\bar{e}=\left[\begin{array}{ccc}
\cos \left(\varphi_{1}\right) & \sin \left(\varphi_{1}\right) & 0 \\
\vdots & \vdots & \vdots \\
\cos \left(\varphi_{m}\right) & \sin \left(\varphi_{m}\right) & 0
\end{array}\right] .
$$

Since the molecular dipoles $\bar{\mu}$ are given in a Cartesian coordinate system, $\bar{\mu}$ has the dimension $3 \times N$. The rotated transition dipoles will have the same dimension. $\bar{A}_{\mu e}(34)$ can be determined by the matrix multiplication $\bar{e} \cdot \hat{\mu}^{\theta}$ and element-wise calculation of the absolute value squared. To calculate the matrices $\bar{f}_{\text {int }}$ and $\bar{f}_{\text {ex }}$, a matrix $\bar{f}$ is set up by introducing the vector $\bar{E}(1 \times n)$, containing the energies

$$
\bar{E}=\left[E_{1}, \ldots, E_{n}\right]
$$

for which we want to calculate the broadening functions (5) and (6). Then the matrix $\bar{f}$ is constructed as the $(N \times n)$ matrix

$$
\bar{f}=\left[\begin{array}{ccc}
f_{1}\left(E_{1}\right) & \ldots & f_{1}\left(E_{n}\right) \\
\vdots & \ddots & \vdots \\
f_{N}\left(E_{1}\right) & \ldots & f_{N}\left(E_{n}\right)
\end{array}\right],
$$


where $f(E)$ is given by (6) in the case of absorption, and by (5) in case of emission. In the absorption case (40) only has to be calculated for one value of $E$, i.e. $E=E_{e x}$, and $\bar{f}$ will therefore be an $(N \times 1)$ vector. The matrix $\bar{f}_{\text {ex }}$ is given by constructing a diagonal matrix with this vector as its diagonal elements. In the case of emission the vector $\bar{E}$ is chosen as

$$
\bar{E}=\left[E_{1}, \ldots, E_{f}\right]
$$

where $E_{1}$ is the lowest detected value of emission and $E_{f}$ is the energy of the cut-off filter. Since this is the upper limit in the integral, there is no need to calculate the emission for higher energies. Trapezoidal integration of the functions $f_{\alpha}$ is used in (40) to obtain a vector giving the the diagonal elements of $\bar{f}_{\text {int }}$ in (33). Finally equations (32) and (33) give the matrices used in the quadratic form (29), which finally gives the fluorescence spectra $\bar{F}$.

\subsection{Parameters}

\begin{tabular}{l|c}
\hline Parameter & Value \\
\hline \hline Electronic couplings: $V_{i, j}$ & $\left(\mathrm{~cm}^{-1}\right)$ \\
Nearest neighbour & \\
B850-B850 & 412 \\
B850-B850 & 308 \\
B850-B800 & 29 \\
B800-B800 & -26 \\
& \\
\hline System-bath coupling: & $100 \mathrm{~cm}^{-1}$ \\
$E_{0}$ & \\
& $\left(\mathrm{~cm}^{-1}\right)$ \\
\hline Gaussian distribution $(\sigma)$ of $\Delta_{j}:$ & 70 \\
B800 & 300 \\
B850 & \\
\hline Lineshape functiton, $\sigma$ of Gaussian & $\left(\mathrm{cm}^{-1}\right)$ \\
$77 \mathrm{~K}$ & \\
B800 & 100 \\
B850 & 140 \\
RT & 145 \\
B800 & 200 \\
B850 &
\end{tabular}

\section{Switching of Configurations Simulated By Rejec- tion Sampling}

The configuration switching effects discussed in the paper are simulated by a rejection sampling algorithm using a uniform proposal distribution and a probability that 
a molecule is given a new energy is given by the probability density function

$$
P(i)=\sum_{\alpha}\left|C_{i, \alpha}\right|^{2} Z\left(\mathcal{E}_{\alpha}\right)
$$

where

$$
Z\left(\mathcal{E}_{\alpha}\right)=\frac{\exp \left(-\mathcal{E}_{\alpha} / k_{B} T\right)}{\sum_{\beta} \exp \left(-\mathcal{E}_{\beta} / k_{B} T\right)}
$$

The proposed values, $z_{n}$, are drawn from a uniform distribution, $z \in U(0,1)$, and accepted if $z_{n}<P(i)$, where $i \in U(1, N), i=1,2,3 \ldots$ Then, the $i$ :th molecular energy is (slightly) changed by drawing a new value of $\Delta_{i}, \Delta \in N\left(\eta, \vartheta^{2}\right)$.

\section{Calculation of Intensity Plots}

To simplify the analysis of a large number of spectra three numbers are chosen to describe each spectrum. These are the excitation modulation depth, $M_{e x}$, emission modulation depth, $M_{e m}$, and the polarization phase shift, $\Delta \varphi$. To calculate these, each spectrum $\bar{F}$ is transformed to the vectors $\bar{I}_{a b s},(1 \times m)$, and $\bar{I}_{e m},(m \times 1)$, according to, [1]:

$$
\bar{I}_{e m}\left(\varphi_{e m}\right)=\int_{\varphi_{1}}^{\varphi_{m}} \bar{F}\left(\varphi_{e m}, \varphi_{e x}\right) \mathrm{d} \varphi_{e x}
$$

and

$$
\bar{I}_{a b s}\left(\varphi_{e x}\right)=\int_{\varphi_{1}}^{\varphi_{m}} \bar{F}\left(\varphi_{e m}, \varphi_{e x}\right) \mathrm{d} \varphi_{e m} .
$$

The integrals above can be calculated using trapezoidal integration. The modulation depth is defined as

$$
M=\frac{I^{\max }-I^{\min }}{I^{\max }+I^{\min }} .
$$

The intensity reaches it maximum value for a certaion polarization angle, $I^{\max }=I\left(\varphi^{\max }\right)$, and the polarization phase shift is defined as, [4]:

$$
\Delta \varphi=\varphi_{e x}^{\max }-\varphi_{e m}^{\max } .
$$

A matrix giving the density for the modulation depth correlation plot points is given by defining $\bar{M},\left(M_{\text {res }} \times M_{r e s}\right)$. This matrix is defined to have $M_{e x}$ on the $x$-axis and $M_{e m}$ on the $y$-axis. For each simulated spectra, $k=M_{e m} \cdot M_{\text {res }}$ and $l=M_{e x} \cdot M_{\text {res }}$ are calculated and rounded up to the nearest integer. This molecular complex is then classified to belong to element $\bar{M}(k, l)$ which value is increased by 1 . Repeating the procedure for all simulated spectra builds up the correlation density.

Standard histogram functions can be used to make intensity plots for $\bar{\Delta} \varphi$.

\section{Illustration of no switching}

Modulation depth correlation plots with no switching of states are shown in the figures S1 and S2. Clearly the calculated modulation depth shows significantly higher $M_{e m}$ values than the experiment. 


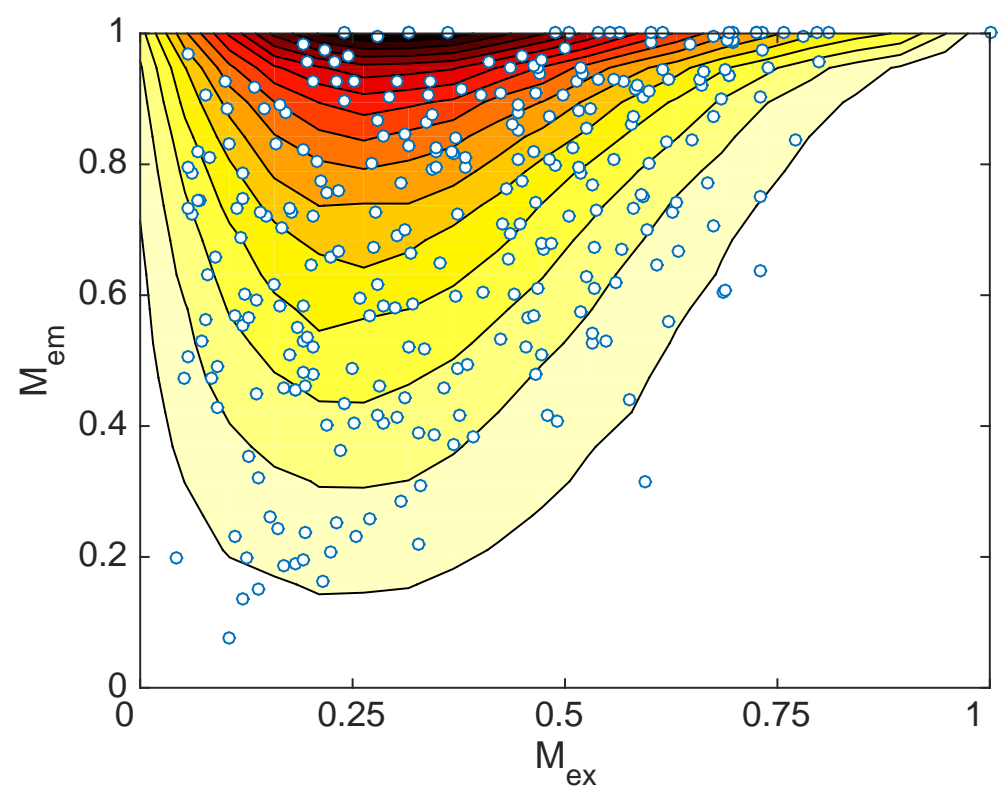

Figure S1: B800 excitation, 77K

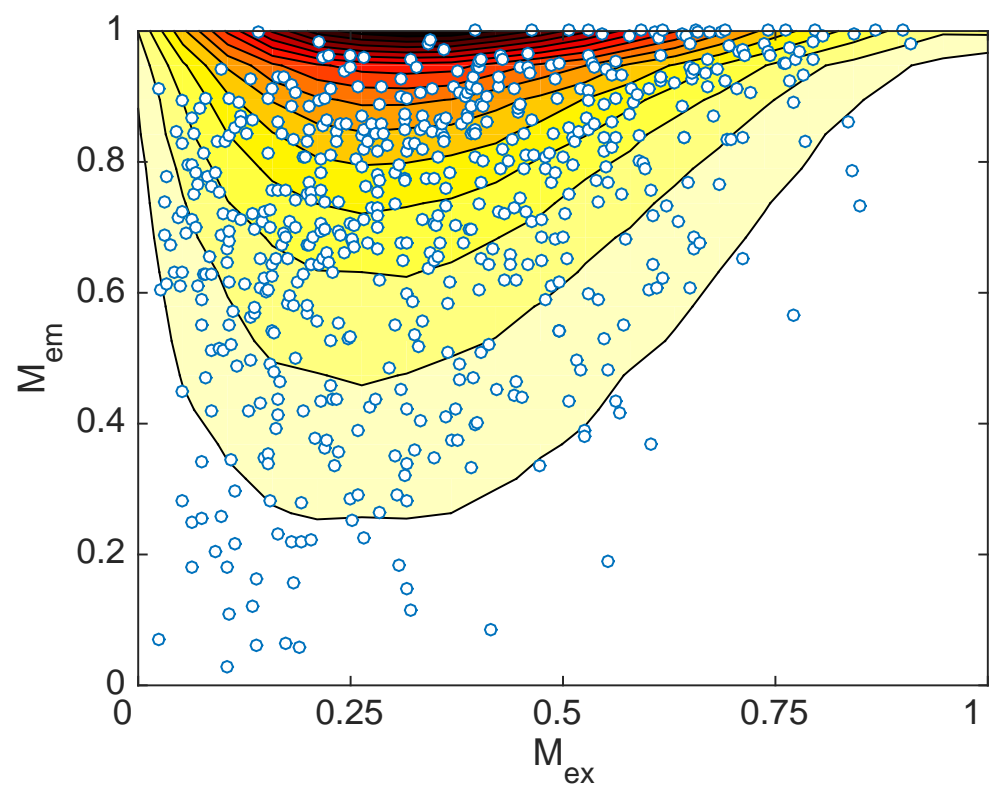

Figure S2: B850 excitation, 77K

\section{References}

[1] Mirzov, O.; Bloem, R.; Hania, P. R.; Thomsson, D.; Lin, H.; Scheblykin I. G. Polarization Portraits of Single Multichromophoric Systems: Visualizing Conformation and Energytransfer. Small 2009, 16, 1877-1878.

[2] Thulstrup, E. W.; Michl, J. Elementary Polarization Spectroscopy. VCH Publishers, 
1989.

[3] Jean, J. M.; Chan, C. K.; Fleming, G. R.; Owens, T. G. Excitation Transport and Trapping on Spectrally Disordered Latticies. Biophys. J. 1989, 56, 1203-1215.

[4] Tubasum, S.; Cogdell, R.; Thomsson, D.; Reus, M.; Scheblykin, I. G.; Pullerits, T. Excitation-Emission Polarization Spectroscopy of Single Light Harvesting Complexes. J. Phys. Chem. B 2011, 115, 4963-4970.

[5] Spano, F. C. Absorption and Emission in Oligo-Phenylene Vinylene Nanoaggregates: The Role of Disorder and Structural Defects. Chem. Phys. 2002, 116, 5877-5891.

[6] Davydov, A .S. Theory of Molecular Excitons. New York, McGraw-Hill, 1962.

[7] Pullerits, T. Exciton States and Relaxation in Molecular Aggregates: Numerical Study of Photosynthetic Light Harvesting. J. Chin. Chem. Soc. 2000, 47, 773-784.

[8] Leegwater, J. A.; Durrant J. R.; Klug D. R. Exciton Equilibration Induced by Phonons: Theory and Applications to PSII Reaction Centers. J. Phys. Chem. B 1997, 101, 7205-7210.

[9] Pullerits, T.; Freiberg, A. Kinetic Model of Primary Energytransfer and Trapping in Photosynthetic Membranes. Biophys. J. 1992, 63, 879-896. 